\begin{tabular}{lc}
\hline & International Journal of Health Services Research and \\
Policy & $\begin{array}{c}\text { Www.dergipark.org.tr/ijhsrp } \\
\text { INESEG }\end{array}$ \\
INTERNATIONAL & e-ISSN: $2602-3482$ \\
ENGINEERING & \\
SCIENCE AND & \\
EDUCATION & \\
GROUP &
\end{tabular}

Research Article

\title{
ADHERENCE TO PROPHYLAXIS IN RELATION TO QUALITY OF LIFE AND ANXIETY LEVEL IN TURKISH PATIENTS WITH SEVERE HAEMOPHILIA A
}

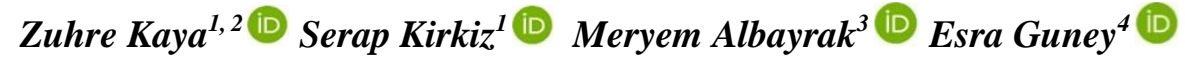 \\ Selin Aytac Eyupoglu 5 (iD) Namik Yasar Ozbek ${ }^{6}$ iD
}

${ }^{1}$ Gazi University, Faculty of Medicine, Department of Paediatrics, Paediatric Haematology Unit, Ankara, Turkey ${ }^{2}$ Gazi University, Department of Paediatrics, Paediatric Haematology Unit, Haemostasis Lab., Ankara, Turkey ${ }^{3}$ Kirikkale University, Faculty of Medicine, Dep. of Paediatrics, Paediatric Hematology Unit, Kırıkkale, Turkey

${ }^{4}$ Gazi University, Faculty of Medicine, Department of Child and Adolescent Psychiatry, Ankara, Turkey

${ }^{5}$ Hacettepe University, Faculty of Medicine, Dep. of Paediatrics, Paediatric Haematology Unit, Ankara, Turkey

${ }^{6}$ University of Health Sciences Ankara, Child Health Diseases Hematology-Oncology Training and Research

Hospital, Ankara, Turkey

Corresponding Author; zuhrekaya@gazi.edu.tr

\begin{abstract}
Bleeding increases anxiety and reduces quality of life (QoL) for patients with severe haemophilia A. Prophylaxis is a therapeutic approach that enhances QoL for these individuals; however, compliance is a major issue. This study investigated adherence to prophylaxis in relation to QoL and anxiety level in patients with severe haemophilia A and in their parents. Forty-three patients with severe haemophilia A were stratified into three groups by age: 12 children aged 2-13 years, 17 adolescents aged 14-21 years, and 14 adults aged >21 years (range, 21-65 years). Quality of life and anxiety level were assessed using the 36-item Short Form Health Survey (SF-36) and the State-Trait Anxiety Inventory (STAI), respectively. Regular prophylaxis $(R P)$ and pharmacokinetic $(P K)$-guided prophylaxis were prescribed for 30 and 13 patients, respectively. All the children ( $n=12$; nine who received $R P$, three who received $P K$-guided prophylaxis) and $10(58.8 \%$ ) of the adolescents (all of whom received PK-guided prophylaxis) were completely adherent to prophylaxis. Seven (41.2\%) of the adolescents and all 14 adults (100\%) were non-adherent to prophylaxis. Compared to findings for the adolescents, anxiety level was higher among the children's parents and among the adult patients ( $p<0.05$ for both). The QoL level was lower for the adult patients than for the parents of child patients and for the adolescent patients ( $p<0.05$ for both). Regarding patients with severe haemophilia A, PKguided prophylaxis for adults and home treatment for small children would enhance adherence to prophylaxis, increase QoL and reduce anxiety levels.
\end{abstract}

Keywords: Prophylaxis, adherence, quality of life, anxiety, haemophilia

Received: May 20, 2020

Accepted: November 3,2020 


\section{Introduction}

Haemophilia is a rare bleeding disorder, which leads to early disability if untreated with factor concentrates on time. For patients with severe haemophilia A, the risk of bleeding increases anxiety and reduces quality of life (QoL). Regular prophylaxis is a therapeutic approach that enhances QoL for these individuals; however, compliance is a major issue [1-5]. In addition to patient compliance, pharmacokinetic (PK) guided prophylaxis would allow optimizing the treatment of individual haemophilia patients [6-10]. Although several studies have evaluated the QoL of patients with severe haemophilia, to the best of our knowledge, no study has yet investigated QoL together with anxiety level in these patients [11-17].

This observational study was aim to analyse adherence to prophylaxis in relation to QoL and anxiety level through questionnaires answered by older patients with severe haemophilia $\mathrm{A}$ and the parents of child patients.

\section{Materials and Methods}

Forty-three patients with severe haemophilia A who were attended in haemophilia camp in 2019 were enrolled in this study. These patients were stratified into three groups by age: 12 children aged 2 13 years, 17 adolescents aged 14-21 years, and 14 adults aged >21 years (range, 21-65 years). Individuals were excluded from the study if they were diagnosed haemophilia B or von Willebrand Disease. Quality of life and anxiety level were assessed in the children's parents and in the adolescent and adult patient groups using the 36-item Short Form Health Survey (SF-36) and the State-Trait Anxiety Inventory (STAI), respectively. The study was approved by the Ethics Committee of Gazi University.

The following patient data were recorded: type of prophylaxis (regular prophylaxis or PK); viral test results (human immunodeficiency virus [HIV], hepatitis B virus [HBV], and hepatitis $\mathrm{C}$ virus $[\mathrm{HCV}]$ ); inhibitor status; location/s and frequency of joint damage; presence of target joints (defined as joints that had sustained bleeding at least three times in the past 6 months); surgery; radiosynovectomy.

Ethical Considerations: This work was carried out by obeying research and ethics rules. Ethical committee consent was obtained from the Ethics Committee of Gazi University, Ankara, Turkey (Date: 28.01.2019, Number: 77).

\subsection{Assessment of Prophylaxis and Patient Activity Status}

Twenty-seven patients without inhibitor were prescribed a regular prophylactic regimen of standard half-life recombinant factor VIII 25-40 IU/kg three times weekly. The remaining 13 patients without inhibitor received PK-guided prophylaxis and were assessed using myPKFIT ${ }^{\circledR}$. Prophylaxis with activated prothrombin complex concentrates was given as 50 to $75 \mathrm{IU} / \mathrm{kg}$ three times weekly in three patients with inhibitor. The following were recorded before the questionnaires were applied: whether the patient had received prophylaxis, whether the patient had experienced vascular access problems, prophylaxis interval/s, adherence to prophylaxis, and where the prophylaxis was administered and by whom. Adherence to prophylaxis was defined as one of two categories in accordance with comparable report [18]. Patients who received $80-100 \%$ of prescribed doses were recorded as "completely adherent" to prophylaxis, and those who received less than $80 \%$ of prescribed doses were 
recorded as "nonadherent." School and work success, and patient activity status, including participation in a sports or exercise program and time spent surfing the Internet, were recorded as well.

\subsection{Study Questionnaires: The 36-Item Short Form Health Survey and the State-Trait Anxiety Inventory}

The SF-36, an internationally standardized, validated and reliable QoL questionnaire, was answered by all adolescent patients, adult patients, and the parents of the child patients. The SF-36 is a self-report instrument that consists of 36 questions in eight domains: physical functioning (PF), role physical (RP), role emotional (RE); general health (GH), vitality (VT), social functioning (SF), mental health (MH), and bodily pain (BP) [16]. The score for each domain depends on the responses to the questions in the questionnaire. Scores range from 0, which indicates the worst possible QoL, to 100, which indicate the best possible QoL.

The STAI is composed of two separate 20-item subscales that measure trait (baseline) (STAI-T) and state (situational) (STAI-S) anxiety [17]. This is a self-report questionnaire that can be administered in an individual format. Specific instructions are provide for each of the S-Anxiety and T-Anxiety subscales, and each of these sections is completed in 10 minutes. The STAI-S evaluates how a person feels in a certain situation and condition, and the STAI-T evaluates how a person feels regardless of a certain situation and condition. The STAI was also completed by all adolescent and adult patients, and by the parents of the child patients. The total score for this questionnaire ranges between 20 and 80 , with higher scores indicating high level of anxiety.

\subsection{Statistical Analysis}

Data were analyzed using the Statistical Package for the Social Sciences (SPSS., Inc., Chicago, IL, USA) version 15.0. Comparisons among group results were performed using the Mann-Whitney $U$ test. Spearman's correlation analysis was used to evaluate for a statistical association between joint damage and SF-36 score. Reliability of data was calculated using Cronbach's alpha analysis. The Cronbach's alpha was 0.7-0.9 and the significance level was set at $p<0.05$.

\section{Results}

The median ages were 6 years for the children's group $(n=12), 17$ years for the adolescent group $(n=17)$, and 34 years for the adult group $(n=14)$. The results of viral screening for HIV, HBV and HCV were negative in all cases. Presence of an inhibitor was detected in three (7.0\%) of the 43 patients, one adolescent (2.3\% of all patients), and two adults (4.7\% of all patients). These individuals had high inhibitor titers (35-95 BU) for more than 10 years. All were on bypassing-agent prophylaxis. Demographic characteristics of the patients in the study are listed in Table 1.

Assessment of joint bleeding revealed 16 patients (37.2\%) with no target joint (i.e., individuals with zero joint bleeds or one bleed only) and 27 (62.8\%) with target joints. Of the 16 individuals with no target joints, 10 children ( $83.4 \%$ of all the children) and four adolescents ( $23.3 \%$ of all adolescents) had a single joint bleed, and two children (the remaining $16.6 \%$ of all children) had no joint bleeding. Of the 27 individuals with target joints, 15 patients ( 10 adolescents [58.4\% of all adolescents] and five adults [35.7\% of all adults]) had $<3$ target joints and 12 patients (three adolescents $[18.3 \%$ of all adolescents] and nine adults [ $64.3 \%$ of all adults] had $\geq 3$ target joints. Eight patients had only a single target joint (five knees [62.5\%], two elbows [25.5\%], one ankle [12.0\%]), seven patients had two target 
joints (knee and elbow in five cases; knee and ankle in two cases), and 12 patients had three or more target joints (knee, ankle and elbow in 11 cases; knee, ankle, elbow, and hip in one case). Nine (33.3\%) of the 27 patients with target joints had undergone surgery, 17 (62.9\%) had undergone radiosynovectomy, and one (3.8\%) had undergone a hip prosthesis procedure [19].

Table 1. Demographic characteristics of the patients $(n=43)$ with severe haemophilia A.

\begin{tabular}{|c|c|}
\hline \multicolumn{2}{|l|}{ Characteristics } \\
\hline Median age (years) & 15 \\
\hline \multirow[t]{2}{*}{ min-max (years) } & $(2-65)$ \\
\hline & $\mathrm{n}(\%)$ \\
\hline \multicolumn{2}{|l|}{ Age groups } \\
\hline 2-13 years & $12(28.0)$ \\
\hline $14-21$ years & $17(39.5)$ \\
\hline$>21$ years & $14(32.5)$ \\
\hline \multicolumn{2}{|l|}{ Inhibitor } \\
\hline Present & $3(6.9)$ \\
\hline Absent & $40(93.1)$ \\
\hline \multicolumn{2}{|l|}{ Prophylaxis } \\
\hline Regular & $30(70.8)$ \\
\hline Pharmacokinetic & $13(30.2)$ \\
\hline \multicolumn{2}{|c|}{ Adherence to prophylaxis } \\
\hline Adherent & $22(51.2)$ \\
\hline Non-adherent & $21(48.8)$ \\
\hline \multicolumn{2}{|l|}{ Home treatment } \\
\hline Yes & $37(86.1)$ \\
\hline No & $6(13.9)$ \\
\hline \multicolumn{2}{|l|}{ Target joint } \\
\hline Yes & $27(62.8)$ \\
\hline No & $16(37.2)$ \\
\hline \multicolumn{2}{|l|}{ Surgery } \\
\hline Yes & $10(23.2)$ \\
\hline No & $33(76.8)$ \\
\hline
\end{tabular}

\subsection{Prophylaxis Status}

All of the child patients (100.0\%; nine on regular prophylaxis and three on PK-guided prophylaxis) and 10 adolescent patients (58.8\%; all on PK-guided prophylaxis) were completely adherent to prophylaxis. Seven (41.2\%) of the adolescent patients and all $14(100 \%)$ of the adult patients were non-adherent to prophylaxis. All 12 children experienced vascular access problems due to their young age. Half of them (all those younger than 7 years of age) were receiving prophylaxis in hospital, whereas the other six were receiving it at home (two older than 10 years doing self-treatment; four 
between 7 and 10 years being treated by their parents). All the adolescent patients were receiving treatments at home, with $15(88.2 \%)$ self-administering and two (11.8\%) being treated by a family member. All the adult patients were self-administering their prophylaxis at home.

\subsection{Activity Status}

Of the 43 total patients, 34 (79.0\%) said they spent less than 6 hours surfing the Internet and nine (21.0\%) reported spending more than 6 hours. Fifteen patients $(34.9 \%)$ said they were exercising regularly, whereas 13 (30.2\%) exercised rarely and 15 (34.9\%) had never exercised more than walking. Nine (64.3\%) of the 14 adult patients expressed that they were reluctant to receive prophylaxis because they had a sedentary lifestyle and did not participate in sports activities.

\subsection{Quality of Life Assessment: 36-Item Short Form Health Survey}

The respective Cronbach's alpha coefficients for the questions evaluating the RE, RP, PF, and BP domains were 0.90, 0.92, 0.78, and 0.96 (Table 2). Regarding the PF, VT, MH, and GH domains, the adolescent group had significantly higher mean scores than the adult group ( $p: 0.01, p: 0.007, p: 0.03$, and $p: 0.02$, respectively) (Table 3 ). The parents of the child patients had a significantly higher mean score for the VT domain than the adult patients ( $p: 0.04)$ (Table 3). The patients on PK prophylaxis had significantly higher mean scores for the $\mathrm{PF}, \mathrm{RP}, \mathrm{MH}, \mathrm{SF}$, and $\mathrm{GH}$ domains than the patients receiving regular prophylaxis ( $p: 0.001, p: 0.03, p: 0.04, p: 0.01$, and $p: 0.02$, respectively) (Table 4$)$. When data for all 43 patients were analyzed, significant negative correlations were detected between number of target joints and the mean scores for the PF ( $r=-0.51 ; p: 0.001), \mathrm{SF}(r=-0.75, p: 0.001), \mathrm{BP}(r=-0.60, p: 0.001)$, and GH $(r=-0.62, p: 0.001)$ domains (Figure 1, Figure 2).

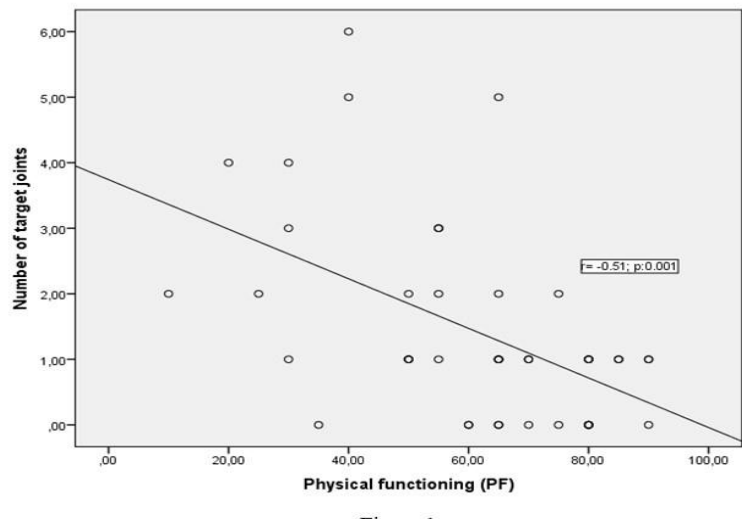

Figure 1a

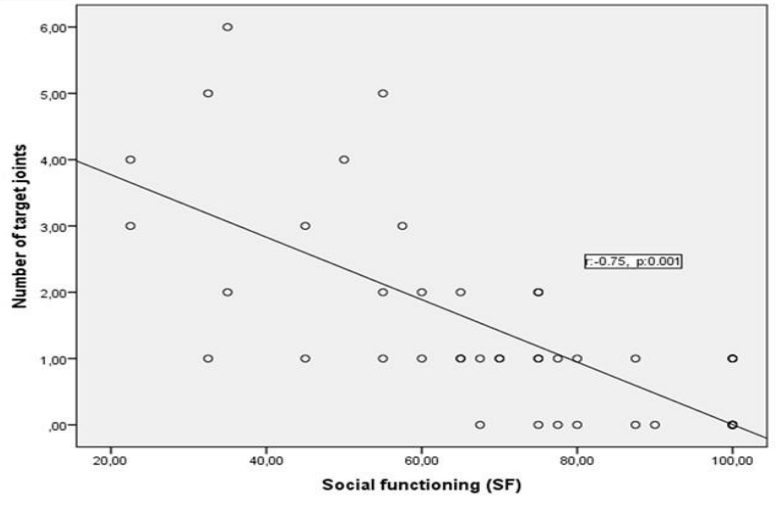

Figure 1b

Figure 1. Correlations between number of target joints and the mean scores for the physical functioning (1a) and social functioning (1b) 
Table 2: Mean scores and internal consistency of quality of life (SF-36) scale and State Trait Anxiety Inventory (STAI) anxiety scale in 43 patients with severe haemophilia A

\begin{tabular}{|c|c|c|c|}
\hline & Items & Mean(SD) & Cronbach $\alpha$ \\
\hline \multicolumn{4}{|l|}{ SF-36 scale } \\
\hline $\mathrm{PF}$ & 10 & $60.8 \pm 20.4$ & 0.78 \\
\hline $\mathrm{RP}$ & 4 & $50.5 \pm 45.4$ & 0.92 \\
\hline RE & 4 & $62.4 \pm 44.5$ & 0.90 \\
\hline VT & 4 & $61.7 \pm 19.6$ & 0.56 \\
\hline MH & 5 & $68.9 \pm 13.5$ & 0.21 \\
\hline SF & 2 & $69.4 \pm 22.9$ & 0.38 \\
\hline BP & 2 & $64.7 \pm 29.6$ & 0.96 \\
\hline GH & 5 & $52.7 \pm 11.4$ & 0.14 \\
\hline Total & & $60.9 \pm 18.9$ & 0.82 \\
\hline \multicolumn{4}{|l|}{ STAI scale } \\
\hline STAI-T & 20 & $35.7 \pm 9.3$ & 0.90 \\
\hline STAI-S & 20 & $40.4 \pm 6.7$ & 0.77 \\
\hline Total & & $38.1 \pm 7.1$ & 0.93 \\
\hline
\end{tabular}

SF-36, Short Form-36; PF, physical functioning; RP, role physical; RE, role emotional; VT, vitality; MH, mental health; SF, social functioning; BP, bodily pain; GH, general health; STAI, State-Trait Anxiety Inventory; STAI-T, STAI-trait; STAI-S, STAI-state.
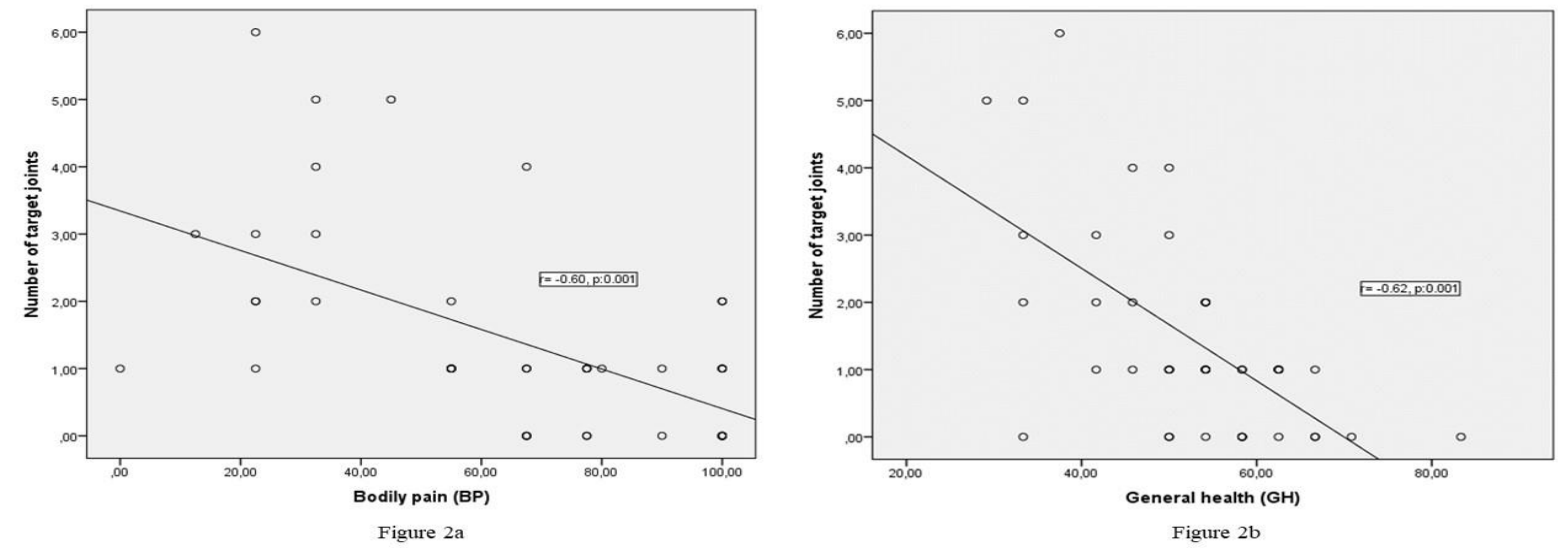

Figure 2. Correlations between number of target joints and the mean scores for the bodily pain (2a) and general health $(2 \mathrm{~b})$ 


\subsection{Anxiety Assessment: State-Trait Anxiety Questionnaire:}

The Cronbach's alpha coefficients for STAI-T and STAI-S were 0.90 and 0.77 , respectively (Table 2). The adult patients had significantly higher scores on the STAI-T than the adolescents and the parents of the child patients ( $p: 0.04$ and $p: 0.04$, respectively), and this reflected higher levels of regret and anxiety among the adult patients. The adolescent patients had significantly lower scores on the STAI-S than the adult group and the children's parents ( $p: 0.04$ and $p: 0.03$, respectively); thus, the adolescents felt comparatively happier and safer (Table 3). The mean STAI-T and STAI-S subscale scores for the patients on PK-guided prophylaxis were not significantly different from those for the patients receiving regular prophylaxis ( $p: 0.51$ and $p: 0.32$, respectively) (Table 4).

Table 3. Comparison of the mean scores of Short Form-36 scale and the State-Trait Anxiety Inventory between the study groups

\begin{tabular}{lllllll}
\hline & Children & Adolescents & Adults & & & \\
& $\mathrm{n}=12$ & $\mathrm{n}=17$ & $\mathrm{n}=14$ & $\mathrm{p}^{*}$ & $\mathrm{p}^{* *}$ & $\mathrm{p}^{* * *}$ \\
& Mean $\pm \mathrm{SD}$ & Mean $\pm \mathrm{SD}$ & Mean $\pm \mathrm{SD}$ & & & \\
\hline SF-36 scale & & & & & & \\
PF & $62.1 \pm 18.6$ & $69.4 \pm 15.1$ & $49.2 \pm 23.1$ & 0.27 & 0.21 & $\mathbf{0 . 0 1}$ \\
RP & $43.7 \pm 45.3$ & $61.7 \pm 43.4$ & $42.8 \pm 48.4$ & 0.36 & 0.86 & 0.37 \\
RE & $69.4 \pm 45.9$ & $66.7 \pm 40.8$ & $50.0 \pm 48.4$ & 0.72 & 0.26 & 0.31 \\
VT & $63.5 \pm 15.8$ & $70.2 \pm 15.3$ & $49.8 \pm 22.1$ & 0.32 & $\mathbf{0 . 0 4}$ & $\mathbf{0 . 0 0 7}$ \\
MH & $68.6 \pm 13.6$ & $73.6 \pm 13.2$ & $63.4 \pm 12.5$ & 0.42 & 0.23 & $\mathbf{0 . 0 3}$ \\
SF & $65.8 \pm 22.7$ & $78.9 \pm 15.3$ & $61.1 \pm 27.5$ & 0.10 & 0.64 & 0.06 \\
BP & $61.2 \pm 32.6$ & $70.0 \pm 22.3$ & $61.2 \pm 35.6$ & 0.48 & 0.96 & 0.58 \\
GH & $51.7 \pm 9.6$ & $59.8 \pm 9.3$ & $44.9 \pm 10.1$ & 0.06 & 0.07 & $\mathbf{0 . 0 2}$ \\
STAI scale & & & & & & \\
STAI-T & $33.7 \pm 10.3$ & $33.8 \pm 8.5$ & $39.5 \pm 8.4$ & 0.94 & $\mathbf{0 . 0 4}$ & $\mathbf{0 . 0 4}$ \\
STAI-S & $41.2 \pm 4.8$ & $36.1 \pm 7.3$ & $43.4 \pm 6.8$ & $\mathbf{0 . 0 4}$ & 0.25 & $\mathbf{0 . 0 3}$
\end{tabular}

SF-36, Short Form-36; PF, physical functioning; RP, role physical; RE, role emotional; VT, vitality; MH, mental health; SF, social functioning; BP, bodily pain; GH, general health; STAI, State-Trait Anxiety Inventory; STAI-T, STAI-trait; STAI-S, STAI-state.* Comparison between children and adolescents; ** Comparison between children and adults;

*** Comparison between adolescents and adults 
Table 4. Comparison of the mean scores of Short Form-36 scale and the State-Trait Anxiety Inventory between the prophylaxis types

\begin{tabular}{clll}
\hline & PK prophylaxis & Regular prophylaxis & \\
& $\mathrm{n}=13$ & $\mathrm{n}=30$ & $\mathrm{p}$ \\
& Mean $\pm \mathrm{SD}$ & Mean $\pm \mathrm{SD}$ & \\
\hline SF-36 scale & & & \\
PF & $70.1 \pm 14.3$ & $49.2 \pm 15.4$ & $\mathbf{0 . 0 0 1}$ \\
RP & $63.5 \pm 43.6$ & $34.2 \pm 43.4$ & $\mathbf{0 . 0 3}$ \\
RE & $69.4 \pm 45.9$ & $66.7 \pm 40.8$ & 0.44 \\
VT & $66.6 \pm 11.8$ & $56.1 \pm 14.3$ & 0.10 \\
MH & $72.6 \pm 11.9$ & $64.2 \pm 14.2$ & $\mathbf{0 . 0 4}$ \\
SF & $77.1 \pm 15.7$ & $59.8 \pm 25.1$ & $\mathbf{0 . 0 1}$ \\
BP & $71.3 \pm 22.5$ & $56.3 \pm 23.3$ & 0.09 \\
GH & $56.2 \pm 11.7$ & $48.2 \pm 9.4$ & $\mathbf{0 . 0 2}$ \\
STAI scale & & & 0.51 \\
STAI-T & $36.9 \pm 7.1$ & $38.4 \pm 7.5$ & 0.32 \\
STAI-S & $37.8 \pm 8.5$ & $40.3 \pm 6.8$ & \\
\hline
\end{tabular}

SF-36, Short Form-36; PF, physical functioning; RP, role physical; RE, role emotional; VT, vitality; MH, mental health; SF, social functioning; BP, bodily pain; GH, general health; STAI, State-Trait Anxiety Inventory; STAI-T, STAI-trait; STAI-S, STAI-state.PK:Pharmacokinetic.

\section{Discussion}

This observational study revealed that adults with haemophilia A were completely non-adherent to prophylaxis, and tended to feel less safe and unhappier than younger haemophilia A patient. The adults also tended to have more severe haemorrhage-related joint damage and feel more anxious in general. The findings also showed that parents of children with haemophilia A were more stressed than adolescent patients and this is likely because of vascular access problems. Our findings indicated that adherence to prophylaxis enhanced QoL and reduced anxiety in adolescents 14-21 years of age. A study from Turkey reported that the rate of adherence to prophylaxis was found to be higher in adolescents than in children and adults [12]. Because adolescents were trained about self-infusions in Haemophilia summer schools. Other results of that particular study suggested that regular prophylaxis led to reduced joint disability and improved QoL in haemophilia patients, which is consistent with our study findings. Another investigation of haemophilia patient from Greece [11] revealed that decreased scores for the PF and energy domains of the SF-36 were in line with the pathological joint score. This is concordant with our finding that number of target joints was negatively correlated with scores for the PF, SF, BP, and GH domains of the SF-36 in our 43 patients with severe haemophilia A. Significantly higher mean scores for the PF, MH, VT, and GH domains in our adolescent patients compared with adult patients indicated better QoL in the younger group. Moreover, the results of the noted Greek study also suggested that affected emotional and mental health (as documented via the SF-36) led to reduced mobility and avoidance of sports activities due to the risk of bleeding [11]. This is supported by our finding that only 
$30 \%$ of our 43 total patients exercised regularly. Similarly, Lehmeier et al. reported that only $35 \%$ of patients with haemophilia were involved in physical activity [20].

In contrast, we observed high rates of adherence to prophylaxis and regular exercise in the child patient group (2-13 years of age), and they had good joint health because prophylaxis was closely monitored by their families; however, the STAI-S results revealed that, compared with adolescent patients, the parents of affected children carried more anxiety due to vascular access problems and lack of on-time treatment at home. We attribute this to families being in a more emotional state because of bleeding risk. Problems related to vascular access increase the anxiety levels of parents of children with haemophilia. We believe that self-administration at home for nearly $90 \%$ of the adolescent patients enhanced their adherence to treatment. This resulted in mostly PK-guided prophylaxis, which improved QoL and reduced anxiety levels for these individuals. Most home support programs have been designed for all haemophilic patients with bleeding, but there is little data on the role these programs play in supporting families of children with haemophilia [9, 21]. A study from Norway revealed that training the mothers of children older than 4 years about vascular access was associated with improved QoL and reduced anxiety level [9]. Likewise, in our study, lower STAI-S score in the adolescent age group compared with the parents of affected children were attributed to the fact that the adolescent patients sought out vascular access training themselves. Most haemophilia centres recommend that factor prophylaxis can be administered at home by parents and patients who receive training from health care professionals. However, Turkey's current national healthcare system does not include such a routine home treatment program. Our results indicate that parents of young children with haemophilia should be trained in self-administration of factor treatment at home, and that this formal training is needed as part of our national health care program.

All adult patients (defined as $>21$ years) was non-adherent to prophylaxis, and these individuals had higher numbers of target joints than the younger groups. Longer period of life with the disease is likely a factor in this; however, our study showed that adherence to prophylaxis decreased with age, as did the number of target joints increased. The literature notes several reasons for marked decrease in adherence to regular prophylaxis, particularly in adults: cost of prophylaxis, inadequate dose, problems with drug supply, increased risk of cardiovascular diseases, and decreased bleeding due to decelerated metabolism making patients believe they do not need regular factor infusion, which subsequently results in increased joint disability [22-24]. In Turkey, prophylaxis treatment is reimbursed unless the dose exceeds 1,500 IU three times a week in patients without inhibitor for haemophilia. This means that individualized prophylaxis is not possible for most patients, which is why only 13 of our patients were able to receive PK-guided prophylaxis. Whereas prophylaxis treatment is reimbursed without dose limitation in Germany and Canada, it may be partially reimbursed or not reimbursed at all depending on state of residence in the United States [10]. Although few studies have measured adherence to PK-guided prophylaxis in all age groups with haemophilia A, we believe that implementing PK-guided prophylaxis for adult patients would enhance adherence to prophylaxis, increase QoL, and reduce levels of anxiety and regret, all of which have been observed in adolescents [6-8].

We believe that a significant amount of the joint disability in our adult patient group may have resulted from inadequate factor dose per body weight because of Turkey's reimbursement limit. This disability, in turn, leads to reduced adherence to treatment and less participation in sports activities. Our findings indicate that such patients spend more time surfing the Internet than younger groups, and as 
such they avoid sports and exercising because of bleeding concerns. This vicious circle results in a progressively less active lifestyle that can initiate and aggravate joint disabilities.

\section{Strengths and Limitations of the study}

There are some strengths and limitations of this study. This is the first study determining the both quality of life using SF-36 and anxiety level using STAI in patients with severe haemophilia A. The possibility of measurement bias is probably low since the medical information of the participants was collected by their physicians. The sample size of the survey is small. However, the response rate was higher than expected. In the most comprehensive review to date on haemophilia patients, nearly $20 \mathrm{QoL}$ questionnaires, including the SF-36, were reported to be valid, reliable, and acceptable for this patient group [25, 26]. In our study, we used the validated SF-36 questionnaire and found that Cronbach's alpha reliability rates were high. In a separate study from Turkey that was conducted using the Turkish version of the Haemophilia-Specific Quality of Life Index in paediatric and adult haemophilia patients, Cronbach's alpha reliability was found to be high; however, quality of life index of this study was reported to be inadequate based on the results of a Western European-type QoL questionnaire [12]. Another strength of this study was our use of PK guided prophylaxis approach that allowed tailoring haemophilia therapy in adolescent's haemophilia patients.

\section{Conclusions}

Our results suggest that PK-guided prophylaxis would enhance adherence to prophylaxis, increase QoL and reduce anxiety levels for adults with severe haemophilia A. The findings also indicate that improved family education about vascular access for home treatment would improve QoL and reduce anxiety for children who have bleeding risk.

Declaration: The authors confirm that they have no interests that might be perceived as posing a conflict or bias.

Ethical Considerations: This work was carried out by obeying research and ethics rules. Ethical committee consent was obtained from the Ethics Committee of Gazi University, Ankara, Turkey (Date: 28.01.2019, Number: 77).

The compliance to Research and Publication Ethics: This work was carried out by obeying research and ethics rules.

\section{References}

[1] Coppola, A., et al., "Prophylaxis in children with hemophilia: evidence based achievements, old and new challenges", Semin Thromb Hemost. 38 (1), 79-94, 2012.

[2] Schrijvers, L.H., Schuurmans, M.J., Fischer, K. "Promoting self-management and adherence during prophylaxis: evidence-based recommendations for haemophilia professionals", Haemophilia. 22 (4), 499-506, 2016.

[3] Garcia-Dasi, M., et al., "Adherence to prophylaxis and quality of life in children and adolescents with severe hemophilia A", Haemophilia. 21(4), 458-464, 2015. 
[4] Krishnan, S., et al., "Adherence to prophylaxis is associated with better outcomes in moderate and severe hemophilia: results of a patient survey", Haemophilia. 21(1), 64-70, 2015

[5] Lock, J., et al., "Adherence to treatment in a Western European paediatric population with hemophilia:reliability and validity of the VERITAS-Pro scale", Haemophilia. 20(5), 616-623, 2014.

[6] Megias-Vericat, J.E., et al., "Bayesian pharmacokinetic guided prophylaxis with recombinant factor VIII in severe or moderate hemophilia A", Thromb Res. 174,151-162, 2019.

[7] Nagao, A., et al., "Clinical outcome in hemophilia A patients undergoing tailoring of prophylaxis based on population based pharmacokinetic dosing", Thromb Res. 173, 79-84, 2019.

[8] Pasca, S., Milan, M., Sarolo, L., Zanon, E. "PK driven prophylaxis versus standard prophylaxis: When a tailored treatment may be a real and achievable cost saving approach in children with severe haemophilia A", Thromb Res. 157, 58-63, 2017.

[9] Von der Lippe, C., et al. "Treatment of hemophilia: A qualitative study of mothers' perspectives”, Pediatr Blood Cancer. 64(1), 121-127, 2017.

[10] Bullinger, M., et al., "Challenges of patient-reported outcome assessment in hemophilia care-a state of the art review", Value Health. 12 (5), 808-820, 2009

[11] Varaklioti, A., et al., "Health-related quality of life and association with arthropathy in Greek patients with haemophilia", Clin Appl Thromb Hemost. 24(5), 815-821, 2018

[12] Mercan, A., et al., "Hemophilia-Specific Quality of Life Index (Haemo-QoL and Haem-A-QoL questionnaires) of children and adults: result of a single center from Turkey", Pediatr Hematol Oncol. 27 (6), 449-461, 2010

[13] Abali, O., et al., "An examination of the symptoms of anxiety and parental attitude in children with haemophilia”, Turk J Med Sci. 44(6), 1087-1090, 2014

[14] Evans, M., Cottrell, D., Shiach, C. "Emotional and behavioural problems and family functioning in children with haemophilia: a cross sectional survey", Haemophilia. 6(6), 682687,2000

[15] Pinto, P.R., et al., "Emotional distress in haemophilia: Factors associated with the presence of anxiety and depression symptoms among adults", Haemophilia. 24(5), e344-e353, 2018

[16] Ware, J.E., Jr, Sherbourne, C.D. "The MOS 36-item short-form health survey (SF 36). I. Conceptual framework and item selection”, Med Care. 30 (6):473-483, 1992

[17] Julian, L.J., "Measures of anxiety. State-Trait Anxiety Inventory (STAI), Beck Anxiety Inventory (BAI), and Hospital Anxiety and Depression Scale-Anxiety (HADS-A)", Arthritis Care Res. 63, 467-472, 2011.

[18] Schrijvers, L.H., et al., "Unraveling adherence to prophylaxis in haemophilia: a patients' perspective", Haemophilia 21(5), 612-621, 2015 
[19] Kaya, Z., et al., "Succesful total hip replacement with sequential administration of bypassing agents in an adolescent boy with hemophilia A and high titer inhibitors", Blood Coagul Fibrinolysis 28 (5),419-422, 2017

[20] Lehmeier, A., et al., "Participation in physical and sportive activities among adult Turkish people with hemophilia A: A single center experience", Turk J Haematol. 35(1), 81-82, 2018

[21] Hanley, J., et al., "Guidelines for the management of acute joint bleeds and chronic synovitis in haemophilia", Haemophilia 23(4), 511-520, 2017

[22] Geerts, E., van de Wiel, H., Tamminga, R. "A pilot study on the effects of the transition of pediatric to adult health care in patients with haemophilia and in their parents: patient and parent worries, parental illness-related distress and health related quality of life", Haemophilia. 14(5), 1007-1013, 2008

[23] Ugur, MC., Kavakli, K. "Prospective evaluation of non-compliant severe haemophilia. Patients", Turk J Hematol. 36(2), 137-138, 2019

[24] Mannucci, P.M., et al., "How I treat age-related morbitidies in elderly persons with haemophilia", Blood. 114 (26):5256-5263, 2009.

[25] Gringeri, A., von Mackensen, S. "Quality of life in haemophilia", Haemophilia. 14(3), 19-25, 2008

[26] Lins, L., Carvalho, F.M. "SF-36 total score as a single measure of health related quality of life: Scoping review", SAGE Open Med. 4: 2050312116671725, 2016. 